\title{
A MODEL-BASED SCHEME FOR ANTICONTROL OF SOME CHAOTIC SYSTEMS
}

\author{
ÖMER MORGÜL \\ Department of Electrical and Electronics Engineering, Bilkent University, \\ 06533, Bilkent, Ankara, Turkey \\ morgul@ee.bilkent.edu.tr
}

Received July 29, 2002; Revised September 23, 2002

\begin{abstract}
We consider a model-based approach for the anticontrol of some continuous time systems. We assume the existence of a chaotic model in an appropriate form. By using a suitable input, we match the dynamics of the controlled system and the chaotic model. We show that controllable systems can be chaotified with the proposed method. We give a procedure to generate such chaotic models. We also apply an observer-based synchronization scheme to compute the required input.
\end{abstract}

Keywords: Chaotic systems; chaos control; chaotization; anticontrol; chaos synchronization.

\section{Introduction}

Recently there has been an increasing interest between scientists from various disciplines on the analysis and control of chaotic behavior in dynamical systems. The literature is quite rich on the subject; an interested reader may consult various survey papers such as [Fradkov \& Evans, 2002; Boccaletti et al., 2000; Gadre \& Varma, 1997; Chen \& Moiola, 1994], to research monographs such as [Kapitaniak, 2000; Chen \& Dong, 1998; Fradkov \& Pogromsky, 1998], and to a bibliography [Chen, 1996].

The development in the field of chaos control was motivated mainly by the seminal paper [Ott et al., 1990], where the term "controlling chaos" was introduced. This work had a strong influence, especially, on the approach of the physics community to the problem of chaos control and is based on variation of certain parameters which affects chaotic behavior. As opposed to this parametric viewpoint approach, classical approach of engineering, and especially systems and control community to the same problem is based on output and state feedback techniques. We utilize this latter approach in the present work.
Similar to classical control problems, various objectives could be defined for the control of chaotic systems. One may investigate the targeting chaotic trajectories to the fixed points, [Shinbrot et al., 1990], or investigate the stabilization of unstable periodic orbits, [Ott et al., 1990], where the main goal is the suppression of chaotic behavior. Another approach would be the opposite, i.e. to retain the chaotic behavior, or even force a regular behavior into a chaotic one, see e.g. [Chen \& Lai, 1996]. This problem is known as "anticontrol", [Schiff et al., 1994], and received considerable interest due to its potential applications in diverse fields, [Brandt \& Chen, 1997; Ditto, 1996; Goldberger, 1994; Yang et al., 1995]. Various feedback schemes, mostly for discrete-time systems are available in the literature for the anticontrol of such systems, see e.g. [Chen \& Lai, 1996, 1998]. In these works, discrete-time systems were considered and the main aim is to change the Lyapunov exponents of the closed-loop sequence by means of a uniformly bounded input sequence.

In this work, we will consider a model-based approach to the anticontrol of some continuoustime systems. We assume the existence of a chaotic model in an appropriate form. Then we try to match 
the dynamics of the system to be controlled with that of the model chaotic system by means of an appropriate control input. We prove that: (i) any controllable linear time-invariant system can be chaotized with an appropriate input, (ii) this approach could be generalized to a class of nonlinear systems. Since our approach relies on the existence of chaotic models in an appropriate form, the question of the existence of such models in arbitrary dimension should be addressed. We propose a simple procedure to generate such chaotic models in arbitrary dimension. Another question we consider is the computability of the required feedback law by using only the available signals. To estimate the states of the system to be controlled, we propose an observer-based synchronization scheme. Under some mild conditions, exponentially fast synchronization may be achieved, and one can use the estimated states to compute the feedback law. We also comment on the robustness of the proposed scheme.

This paper is organized as follows. In the next section we define the problems considered in this paper and present some developments which will be used in the sequel. In the third section, we propose an anticontrol scheme for linear systems, and then generalize it to a class of nonlinear systems. In the following section we propose an observer-based synchronization scheme to estimate the states of the system to be controlled. In the fifth section, we propose a simple way to generate the model chaotic systems for arbitrary dimension. In the following section we present some simulation results. Finally we give some concluding remarks.

\section{Problem Statement}

We will first consider the linear systems. Consider the system given below:

$$
\dot{x}=A x+B u, \quad y=C x,
$$

where $x \in \mathbf{R}^{n}, A \in \mathbf{R}^{n \times n}$ is a constant matrix, $B, C^{T} \in \mathbf{R}^{n}$ are constant vectors, here superscript $T$ denotes transpose, $u$ is the (scalar) control input and $y$ is the (scalar) output, which is assumed to be measurable. For this system, we pose the following problems:

Problem 1. Find a feedback law $u=g(x)$, where $g: \mathbf{R}^{n} \rightarrow \mathbf{R}$ is an appropriate function, such that the resulting closed-loop system exhibits chaotic behavior.
Problem 2. Assume that the feedback law $u=$ $g(x)$, which solves Problem 1, cannot be computed by using the output $y$ alone. Find an approximate control law $u(t)=\hat{u}(t)$, which can be computed by using output, such that $\|\hat{u}(t)-g(x(t))\| \rightarrow 0$ as $t \rightarrow \infty$; here $x(t)$ is the solution of $(1)$, and $\|\cdot\|$ denotes any norm in $\mathbf{R}^{n}$.

A solution to Problem 1 will be provided in the next section. Later we will present an observerbased scheme for Problem 2. In this approach, the output $y$ will be used as a synchronization signal, and an observer-based synchronization scheme will be used to estimate the states $x$ of (1), see e.g. [Morgül \& Solak, 1996, 1997]. These estimates then will be used to obtain an approximation of the control law $u=g(x)$.

To simplify the analysis, we will first transform the system given by (1) into an appropriate canonical form. Let us define the following matrix:

$$
Q_{c}=\left(\begin{array}{lll}
A^{n-1} B & A^{n-2} B \cdots A B \quad B
\end{array}\right)
$$

It is well known that the system given by (1) is controllable (i.e. any state $x_{0} \in \mathbf{R}^{n}$ can be steered to any state $x_{1} \in \mathbf{R}^{n}$ with an appropriate control input $u$ ) if and only if $\operatorname{rank}\left(Q_{c}\right)=n$, see e.g. [Kailath, 1980]. We will assume that this condition holds, hence $Q_{c}$ is assumed to be invertible.

Let $p(\lambda)$ be the characteristic polynomial of $A$ given by (1), as follows:

$$
\begin{aligned}
p(\lambda) & =\operatorname{det}(\lambda I-A) \\
& =\lambda^{n}+\alpha_{1} \lambda^{n-1}+\cdots+\alpha_{n-1} \lambda+\alpha_{n} .
\end{aligned}
$$

Now, let us define the vectors $u_{1}=\left(1 \alpha_{1} \cdots \alpha_{n-1}\right)^{T}$, $u_{2}=\left(\begin{array}{lllll}0 & 1 & \alpha_{1} & \cdots & \alpha_{n-2}\end{array}\right)^{T}, \ldots, u_{n}=\left(\begin{array}{llll}0 & 0 & \cdots & 1\end{array}\right)^{T}$, and define the matrices $U=\left(u_{1} u_{2} \cdots u_{n}\right), R=$ $\left(Q_{c} U\right)^{-1}$. By using the coordinate transformation $z=R x,(1)$ can be transformed into the following form:

$$
\dot{z}=\hat{A} z+\hat{B} u, \quad y=\hat{C} z
$$

where $z=\left(z_{1} z_{2} \cdots z_{n}\right)^{T}, \hat{A}=R A R^{-1}, \hat{B}=R B$, $\hat{C}=C R^{-1}$. After straightforward calculations and by using Cayley-Hamilton theorem (i.e. $p(A)=0$, where $p(\cdot)$ is given by $(3))$, it can be shown that $\hat{A}$ 
and $\hat{B}$ have the following form:

$$
\begin{aligned}
\hat{A} & =\left(\begin{array}{ccccc}
0 & 1 & 0 & \cdots & 0 \\
0 & 0 & 1 & \cdots & 0 \\
& & \vdots & & \\
0 & 0 & 0 & \cdots & 1 \\
-\alpha_{n} & -\alpha_{n-1} & -\alpha_{n-2} & \cdots & -\alpha_{1}
\end{array}\right), \\
B & =\left(\begin{array}{l}
0 \\
0 \\
0 \\
0 \\
1
\end{array}\right) .
\end{aligned}
$$

\section{An Anti-Control Scheme}

We assume the existence of a chaotic system which has the following form ( for $n \geq 3$ )

$$
\begin{aligned}
\dot{w}_{1} & =w_{2} \\
\dot{w}_{2} & =w_{3} \\
& \vdots \\
\dot{w}_{n-1} & =w_{n} \\
\dot{w}_{n} & =f\left(w_{1}, w_{2}, \ldots, w_{n}\right)
\end{aligned}
$$

where $f: \mathbf{R}^{n} \rightarrow \mathbf{R}$ is an appropriate function. For $n=3$ there are many chaotic systems proposed in the literature which has the form given above, see e.g. [Morgül \& Solak, 1996, 1997]. In fact, many chaotic electronic oscillators proposed in the literature, including the well-known Chua's oscillator, are either in this form, or could be transformed into this form. In Sec. 5, we will present a simple scheme to generate chaotic systems of this form for $n>3$.

Our anti-control scheme is based on matching the system given by (4) with the model chaotic system given by (6) by using an appropriate control input $u$. Note that (6) could be rewritten as

$$
\dot{w}=\hat{A} w+\hat{B} h(w),
$$

where $w=\left(w_{1} w_{2} \cdots w_{n}\right)^{T}$, and

$h(w)=f(w)+\alpha_{1} w_{n}+\alpha_{2} w_{n-1}+\cdots+\alpha_{n} w_{1}$.

Hence, by choosing $u$ as:

$$
\begin{aligned}
u=h(z)= & f(z)+\alpha_{1} z_{n} \\
& +\alpha_{2} z_{n-1}+\cdots+\alpha_{n} z_{1},
\end{aligned}
$$

we can transform (4) into the chaotic system given by (6).
The approach given above can also be applied to a class of nonlinear systems. Let us assume that the system to be controlled is given as

$$
\dot{x}=A(x)+B(x) u, \quad y=C(x),
$$

where $A, B: \mathbf{R}^{n} \rightarrow \mathbf{R}^{n}$ and $C: \mathbf{R}^{n} \rightarrow \mathbf{R}$ are appropriate functions, $u$ and $y$ are control input and measurement outputs, which are scalars.

We assume that there exists a coordinate change $z=T(x)$, where $T: \mathbf{R}^{n} \rightarrow \mathbf{R}^{n}$ is an appropriate function, which transforms (10) into the following form

$$
\dot{z}=\hat{A} z+\hat{B}(\gamma(z)+\beta(z) u), \quad y=\hat{C}(z),
$$

where $\hat{A}, \hat{B}$ are as given in $(5), \alpha_{i}, i=1, \ldots, n$ are appropriate constants, and $\gamma, \beta, \hat{C}: \mathbf{R}^{n} \rightarrow$ $\mathbf{R}$ are appropriate functions. For the existence and construction of such a transformation, see e.g. [Vidyasagar, 1993; Isidori, 1995]. We note that the terms multiplying $\alpha_{i}$ in (11) could be included in $\gamma(z)$.

By using the control law

$$
u=\frac{h(z)-\gamma(z)}{\beta(z)},
$$

where $h(\cdot)$ is given by $(8)$, we can match the dynamics of (11) with that of the model chaotic system given by $(7)$, provided that $\beta(z) \neq 0$. This requirement is natural, since otherwise the control input $u$ has no effect on the system dynamics, see (11).

The approach given above could be extended to second order systems as well. In such a case our model chaotic systems will be a forced chaotic oscillator, e.g. forced Duffing equation, which has the following form

$$
\begin{aligned}
& \dot{w}_{1}=w_{2} \\
& \dot{w}_{2}=f\left(w_{1}, w_{2}\right)+r(t)
\end{aligned}
$$

where $r(t)$ is a special (scalar) forcing term to guarantee chaotic behavior. In this case, the control laws given by (9) and (12) should be replaced by $u=h(z)+r(t)$, and $u=(h(z)+r(t)-\gamma(z) / \beta(z)$, respectively.

The results presented in this section can be summarized as follows

(i) Any controllable linear (single input, $n \geq 2$ ) system can be chaotized with an appropriate control law.

(ii) Any nonlinear (single input, $n \geq 2$ ) system which could be transformed into the form (11) can be chaotized with an appropriate control law provided that $\beta(z) \neq 0$. 


\section{Observer-Based Implementation}

To implement the control laws (9) or (12), the state vector $z$ should be available. In most of the cases, the available output signal $y$ has lower dimension, which is a scalar in our case, and is not sufficient to compute the necessary control input $u$. In such cases, an approximation of $u$ might be appropriate.

To elaborate further, let us consider the linear system given by (4). To compute the control input given by $u$, if $z$ is not available, a natural choice would be to use an estimate of $z$. This could be done by using an observer-based synchronization scheme, see e.g. [Morgül \& Solak, 1996, 1997]. Let us consider the following observer-based synchronization scheme for the system given by (4)

$$
\dot{\hat{z}}=\hat{A} \hat{z}+\hat{B} u+K(y-\hat{y}), \quad \hat{y}=\hat{C} \hat{z},
$$

where $\hat{z} \in \mathbf{R}^{n}, K \in \mathbf{R}^{n}$ is a gain vector to be determined. Let us define the error in synchronization as $e=z-\hat{z}$. By using (4) and (14) we obtain

$$
\dot{e}=(\hat{A}-K \hat{C}) e .
$$

Hence, if $A_{c}=\hat{A}-K \hat{C}$ is a stable matrix, then we have $\|e(t)\| \rightarrow 0$ as $t \rightarrow \infty$; moreover this decay is exponential. Existence of such a vector $K$ is guaranteed if the system given by (4) is observable. More precisely, let us define the following observability matrix

$$
Q_{o}=\left(\begin{array}{c}
C \\
C A \\
\vdots \\
C A^{n-1}
\end{array}\right) .
$$

It is well-known that if $\operatorname{rank}\left(Q_{o}\right)=n$, then there exists a $K$ such that the matrix $A_{c}$ is stable, hence the solutions of (15) satisfy:

$$
\|e(t)\| \leq M e^{-\delta t}\|e(0)\|,
$$

for some $M>0, \delta>0$; moreover the decay rate $\delta$ can be adjusted arbitrarily, see e.g. [Kailath, 1980; Morgül \& Solak, 1997]. Also note that this observability condition is sufficient in many observerbased synchronization schemes, see [Morgül, 1999a]. Based on the estimate $\hat{z}$ of $z$, a natural approximation of $u$ given by (9) is $u=h(\hat{z})$. To see the effect of this approximation, assume that $h: \mathbf{R}^{n} \rightarrow \mathbf{R}$ is a Lipschitz function, i.e. the following holds for some $l>0$

$$
\|h(z)-h(\hat{z})\| \leq l\|z-\hat{z}\| .
$$

Now, assume that we use $u=h(\hat{z})$ in (4). Then, the latter becomes

$$
\dot{z}=\hat{A} z+\hat{B} h(\hat{z})=\hat{A} z+\hat{B} h(z)+e_{c}(t),
$$

where $e_{c}(t)$ is an error term which satisfies

$\left\|e_{c}(t)\right\|=\|\hat{B}(h(\hat{z})-h(z))\| \leq l M e^{-\delta t}\|e(0)\|$,

see (17) and (18). We note that the basic idea presented above is similar to the observer-based control of chaos presented in [Solak et al., 2001]. Since the error term decays to zero exponentially fast, we expect that the behavior of (19) and (7) be qualitatively similar, provided that the chaotic behavior of (7) is structurally stable. If the chaotic solution of (7) is globally attractive, then since $e_{c}(t)$ decays to zero exponentially fast, the solutions of (19) will eventually converge to the chaotic solutions of (7). If the chaotic solutions of (7) are only locally attractive, let us assume that for some $\varepsilon>0$, the behaviors of (19) and (7) are qualitatively similar, provided that $\left\|e_{c}(t)\right\| \leq \varepsilon$. We will call this assumption as the structural stability assumption, see e.g. [Fradkov \& Pogromsky, 1998]. From (20) it easily follows that this condition is satisfied for $\|e(0)\| \leq \varepsilon / l M$. Hence, if initial error is sufficiently small, then the behaviors of (19) and (7) are qualitatively similar under the structural stability assumption given above. On the other hand, assume that $\|e(0)\| \leq R$ for some $R>0$. From (20) it follows that $\left\|e_{c}(t)\right\| \leq \varepsilon$ for $t \geq T=1 / \delta \ln (l M R / \varepsilon)$. Hence we could use a switching law to generate $u$ as follows:

$$
u=\left\{\begin{array}{cc}
0 & t<T \\
h(\hat{z}) & t \geq T
\end{array}\right.
$$

For the system given by (11), the observer-based control law given above may be applied, provided that

(i) $\hat{y}=\hat{C} \hat{z}$, where $\hat{C}=\left(\begin{array}{llll}1 & 0 & \cdots & 0\end{array}\right)^{T}$.

(ii) $\beta(z), \gamma(z)$ and $(h(z)-\gamma(z)) / \beta(z)$ are Lipschitz functions.

If these conditions are satisfied, then the related observer-based synchronization scheme is given as follows

$$
\begin{aligned}
& \dot{\hat{z}}=\hat{A} \hat{z}+\hat{B}(\gamma(\hat{z})+\beta(\hat{z}) u)+K(y-\hat{y}), \\
& \hat{y}=\hat{C} \hat{z},
\end{aligned}
$$

For the existence and computation of an approximate gain vector $K$, see e.g. [Morgül \& Solak, 1996, 1997]. The control input $u$ can be chosen as $u=(h(\hat{z})-\gamma(\hat{z})) / \beta(\hat{z})$. 
Remark 1. The requirement that various functions be Lipschitz may seem to be restrictive. Note that any differentiable function is locally Lipschitz in any bounded domain. Hence, if the solutions remain in a bounded set, which is the case for chaotic systems, then this requirement is satisfied provided that the corresponding functions are differentiable.

Remark 2. We note that the control scheme presented in this work is robust with respect to noise and parameter mismatch under certain conditions. Such effects could be included in the system dynamics by adding a perturbation term to the system equations, similar to $e_{c}(t)$ in (19). This extra perturbation term will be proportional to noise and parameter mismatch magnitudes under certain conditions. Hence this perturbation term will be small provided that the parameter mismatch and noise magnitudes are small. Therefore such a perturbation will not affect the chaotization scheme presented above, provided that the noise and parameter mismatch magnitudes are small. We also note that due to exponential stability, observerbased implementation presented in this work is also robust with respect to noise and parameter mismatch, see e.g. [Morgül \& Feki, 1997; Morgül \& Solak, 1996, 1997; Morgül et al., 2002] for detailed analysis.

\section{Model Chaotic Systems}

Our control scheme is based on the existence of model chaotic systems which has the form given in (6). For $n=3$, such chaotic systems are abundant in the literature. In fact, all Lur'e type systems, which cover most of the electronic chaotic oscillators proposed in the literature including the well-known Chua's oscillator, can be transformed into this form. Some systems, which are not in this structure (e.g. Rössler system), may be transformed into this form, see e.g. [Morgül \& Solak, 1997]. As an example, in our simulations we will use the following system

$$
\begin{aligned}
& \dot{w}_{1}=w_{2} \\
& \dot{w}_{2}=w_{3} \\
& \dot{w}_{3}=-b_{2} w_{3}-b_{1} w_{2}-b_{0} w_{1}-w_{1}^{2}
\end{aligned}
$$

This system exhibits chaotic behavior for a certain range of parameters $b_{i}$, see [Genesio \& Tesi, 1992; Morgül, 1999b].

To generate chaotic systems for $n>3$ which has the form of (6), let us consider the case $n=3$, which is repeated below for convenience

$$
\begin{aligned}
& \dot{w}_{1}=w_{2} \\
& \dot{w}_{2}=w_{3} \\
& \dot{w}_{3}=f\left(w_{1}, w_{2}, w_{3}\right)
\end{aligned}
$$

By defining $w=w_{1}$, and noting that $w_{2}=\dot{w}$, $w_{3}=\ddot{w}$, and by using (24), we obtain the following scalar equation

$$
w^{(3)}-f(w, \dot{w}, \ddot{w})=0 .
$$

Obviously, (25) and (24) are equivalent through the transformation given above. Now let us consider the following higher dimensional system

$$
\begin{aligned}
& \dot{w}_{1}=w_{2} \\
& \dot{w}_{2}=w_{3} \\
& \dot{w}_{3}=f\left(w_{1}, w_{2}, w_{3}\right)+w_{4} \\
& \dot{w}_{4}=-\alpha w_{4}
\end{aligned}
$$

where $\alpha>0$ is an arbitrary constant. Note that $w_{4}(t)=w_{4}(0) e^{-\alpha t} \rightarrow 0$ as $t \rightarrow \infty$. Hence asymptotically, (24) and the first three equations of (26) are the same. Therefore, if (24) has a globally attractive chaotic solution, so does (26). On the other hand, if (24) has only locally attractive chaotic solution, which is structurally stable in the sense given before, then so does (26), provided that $\left|w_{4}(0)\right|$ is sufficiently small.

To transform the system given by (26) into the form given by (6), first note that from the third equation in (26) we have $w_{4}=\dot{w}_{3}-f\left(w_{1}, w_{2}, w_{3}\right)$. By defining $w=w_{1}$, and noting that $w_{2}=\dot{w}, w_{3}=$ $\ddot{w}$, and using the last equation in (26) we obtain

$\frac{d}{d t}\left(w^{(3)}-f(w, \dot{w}, \ddot{w})\right)+\alpha\left(w^{(3)}-f(w, \dot{w}, \ddot{w})\right)=0$,

which could be rewritten as

$$
w^{(4)}=F\left(w, \dot{w}, \ddot{w}, w^{(3)}\right),
$$

where

$$
\begin{aligned}
F\left(w, \dot{w}, \ddot{w}, w^{(3)}\right)= & \frac{d}{d t}(f(w, \dot{w}, \ddot{w}))-\alpha\left(w^{(3)}\right. \\
& -f(w, \dot{w}, \ddot{w})) .
\end{aligned}
$$

Naturally, here we assume that $f$ is a differentiable function. Obviously, (28) is equivalent to (26). By using standard change of variables $w_{1}=w, w_{2}=\dot{w}$, $w_{3}=\ddot{w}, w_{4}=w^{(3)}$, we can rewrite $(28)$ as

$$
\begin{aligned}
& \dot{w}_{1}=w_{2} \\
& \dot{w}_{2}=w_{3} \\
& \dot{w}_{3}=w_{4} \\
& \dot{w}_{4}=F\left(w_{1}, w_{2}, w_{3}, w_{4}\right)
\end{aligned}
$$


which has the form of (6) for $n=4$. Obviously this procedure can be extended to arbitrary dimension, provided that $f$ is sufficiently smooth.

Remark 3. With the procedure outlined above, given a chaotic system of the form (6) with dimension $n$, we can construct higher dimensional chaotic systems of the same form. These systems will have a chaotic attractor, which is qualitatively similar to that of the lower dimensional model system. Hence, from mathematical point of view, these systems will not be more interesting than the original lower dimensional models. Our aim in this section is to show the existence of chaotic systems of the form given by (6) for arbitrary dimension, and the procedure presented above provides one such system. Obviously, for the control scheme presented in Sec. 3, any chaotic system which has the form (6) could be used.

\section{Simulation Results}

As a simulation example, we consider a single link robot arm with a flexible joint. Such a system consists of a single robot arm (link) whose positioning is controlled by a motor and the coupling between the motor shaft and the link has some flexibility. The equations of motion for such a system is given by

$$
\begin{gathered}
\ddot{q}_{1}+\sin q_{1}+\left(q_{1}-q_{2}\right)=0, \\
\ddot{q}_{2}-\left(q_{1}-q_{2}\right)=u,
\end{gathered}
$$

where $u$ is the control torque applied to the motor shaft. For simplicity we assumed unit values for various coefficients. For a detailed explanation of the model and coefficients, see [Vidyasagar, 1993, p. 435]. Assume that the link angle $q_{1}$ is measurable, which is realistic. By using the coordinates $x_{1}=q_{1}, x_{2}=\dot{q}_{1}, x_{3}=q_{2}, x_{4}=\dot{q}_{2}$, this system can be rewritten as

$$
\begin{aligned}
\dot{x}_{1} & =x_{2} \\
\dot{x}_{2} & =-\sin x_{1}-\left(x_{1}-x_{3}\right) \\
\dot{x}_{3} & =x_{4} \\
\dot{x}_{4} & =x_{1}-x_{3}+u \\
y & =x_{1}
\end{aligned}
$$

This system is in the form given by (10). By using the coordinate transformation

$$
\begin{aligned}
& z_{1}=x_{1} \\
& z_{2}=x_{2} \\
& z_{3}=-\sin x_{1}-\left(x_{1}-x_{3}\right) \\
& z_{4}=-x_{2} \cos x_{2}-\left(x_{2}-x_{4}\right)
\end{aligned}
$$

this system is transformed into the following form

$$
\begin{aligned}
\dot{z}_{1} & =z_{2} \\
\dot{z}_{2} & =z_{3} \\
\dot{z}_{3} & =z_{4} \\
\dot{z}_{4} & =\gamma(z)+u \\
y & =z_{1}
\end{aligned}
$$

where

$$
\begin{aligned}
\gamma(z)= & \sin z_{1}\left(z_{2}^{2}+\cos z_{1}+1\right) \\
& -\left(z_{3}+\sin z_{1}\right)\left(2+\cos z_{1}\right) .
\end{aligned}
$$

Note that the system given by (35) is in the form given by (11) with $\beta(z)=1, \gamma(z)$ as given by (36) and $y=\hat{C} z$ with $\hat{C}=\left(\begin{array}{llll}1 & 0 & 0 & 0\end{array}\right)^{T}$. Also note that this transformation is globally invertible. For the construction of this transformation and other details, see [Vidyasagar, 1993, p. 437].

For our model chaotic system, we use the system given by (23). By using the approach given in previous section, and using (29) with $\alpha=1$, we obtain a four dimensional chaotic system given by (30) with

$$
\begin{aligned}
F(w)= & -\left(b_{2}+1\right) w_{4}-\left(b_{1}+b_{2}\right) w_{3} \\
& -\left(b_{0}+b_{1}+2 w_{1}\right) w_{2}-b_{0} w_{1}-w_{1}^{2} .
\end{aligned}
$$

Hence an appropriate control input for the system (35) is

$$
u=F(z)-\gamma(z),
$$

where $F$ and $\gamma$ are given by (37) and (36), respectively. Since only $z_{1}$ is measurable, the control law given above is not computable by using measurements. To estimate the states, we may use an observer-based synchronization scheme as given below

$$
\begin{aligned}
& \dot{\hat{z}}_{1}=\hat{z}_{2}+k_{1}\left(z_{1}-\hat{z}_{1}\right) \\
& \dot{\hat{z}}_{2}=\hat{z}_{3}+k_{2}\left(z_{1}-\hat{z}_{1}\right) \\
& \dot{\hat{z}}_{3}=\hat{z}_{4}+k_{3}\left(z_{1}-\hat{z}_{1}\right) \\
& \dot{\hat{z}}_{4}=\gamma(\hat{z})+k_{4}\left(z_{1}-\hat{z}_{1}\right)+u
\end{aligned}
$$

where $k_{1}, \ldots, k_{4}$ are the gains to be determined. By using the techniques presented in [Morgül \& Solak, 1996, 1997], one can find appropriate gains so that the error $e=z-\hat{z}$ satisfies (17) provided that $\gamma$ is Lipschitz. Note that the existence of such gains are guaranteed if the solutions of (35) and (39) are 


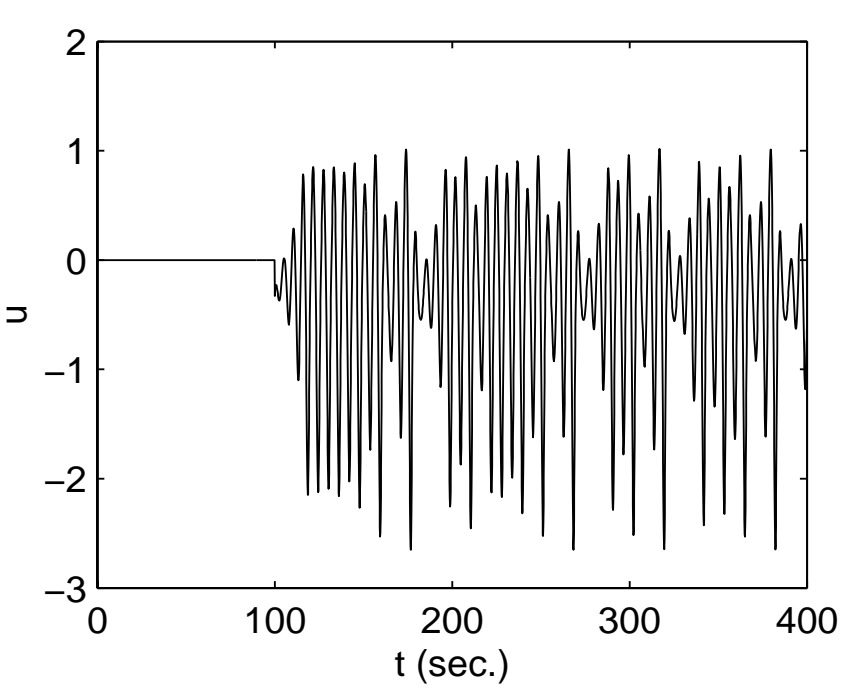

(a)

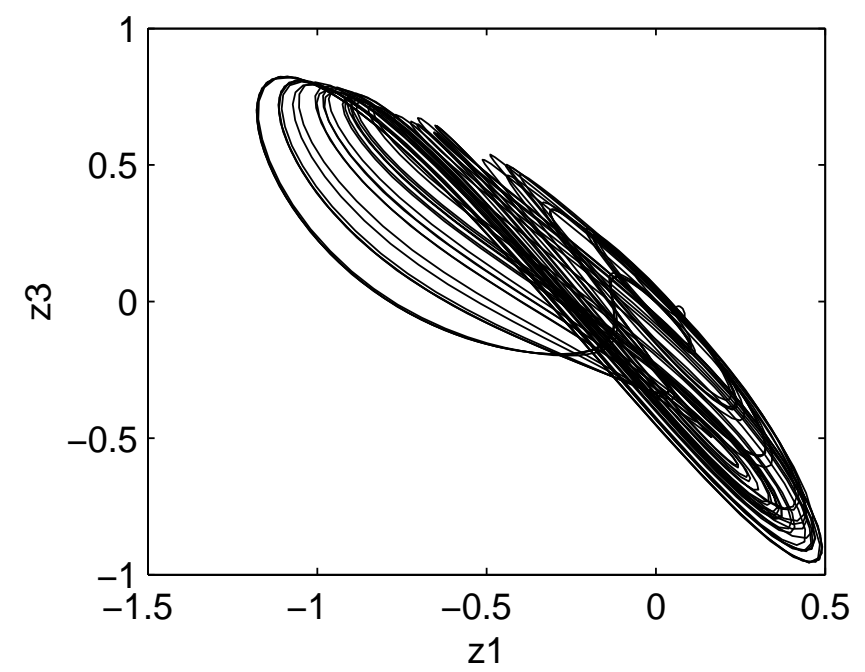

(c)

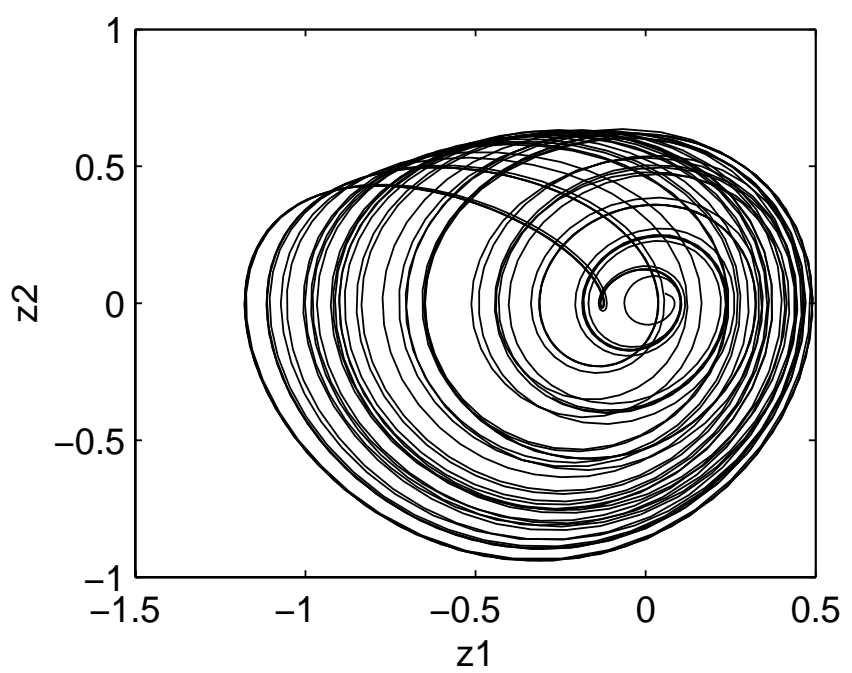

(b)

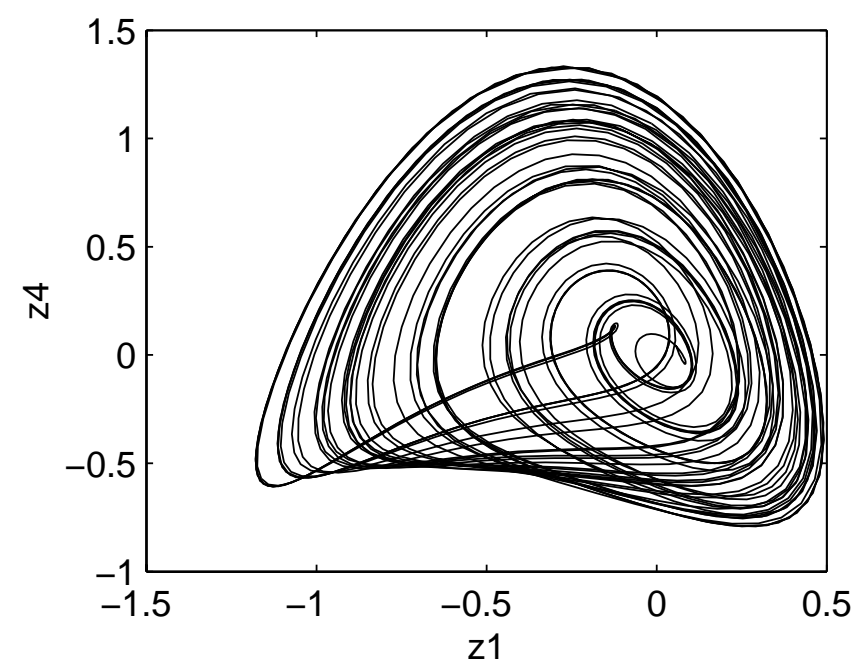

(d)

Fig. 1. Simulation result for flexible beam: ideal case, (a) control input $u$ (applied for $t \geq 100$ ), (b) $z_{1}$ versus $z_{2}$, (c) $z_{1}$ versus $z_{3}$, (d) $z_{1}$ versus $z_{4}$.

bounded, see Remark 1. In this case, the control input is chosen as

$$
u=F(\hat{z})-\gamma(\hat{z}),
$$

In the simulations we choose $b_{0}=1, b_{1}=1.18$, $b_{2}=0.4$, for which (23) exhibits chaotic behavior, see [Genesio \& Tesi, 1992; Morgül, 1999b]. For the observer gains, we use $k_{1}=8, k_{2}=24, k_{3}=32$, $k_{4}=16$. In this case the eigenvalues of $A_{c}=\hat{A}-K \hat{C}$ are set to -2 .

In the first set of simulations, we used (35) and (39) together with the parameter set given above. To see the effect of our control scheme, the control input is applied for $t \geq 100$. The resulting control input $u$ is shown in Fig. 1(a). To exhibit the chaotic behavior, $z_{1}$ versus $z_{2}, z_{3}$ and $z_{4}$ are shown in Figs. 1(b)-1(d), respectively. These figures are plotted for $t \geq 100$.

In the second set of simulations, we considered some nonidealities which may arise due to parameter mismatch and noise. In particular, to see the effect of parameter mismatch, we used $\left(1+r_{2}\right) \gamma(\hat{z})$ in (39) and $u=\left(1+r_{1}\right) F(\hat{z})-\left(1+r_{2}\right) \gamma(\hat{z})$ in (35) and (39); here $r_{1}$ and $r_{2}$ represents the uncertainties in the chaotic model and the system to be controlled, respectively. Moreover, to see the effect of noise, we used $z_{1}+n$ in (39) instead of $z_{1}$; here $n$ is a noise signal distributed in the interval $[-m, m]$. With the parameters given above, and with $r_{1}=0.01, r_{2}=0.1, m=0.1$, we simulated 


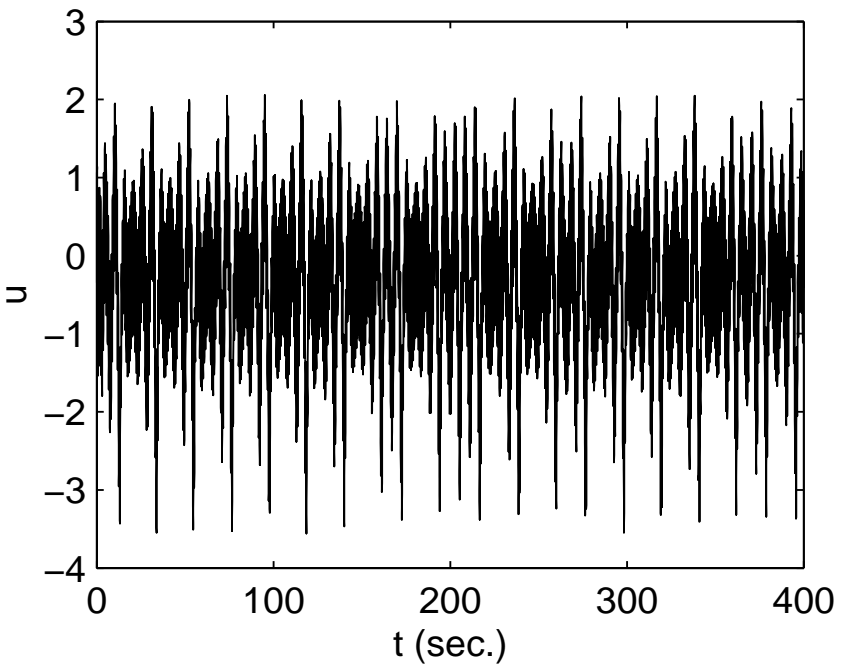

(a)

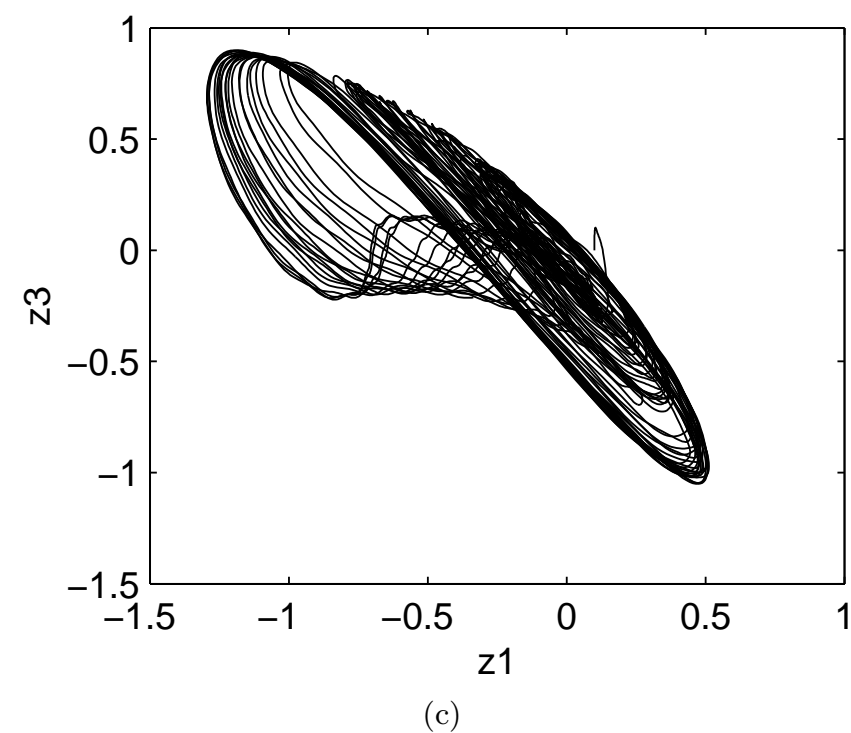

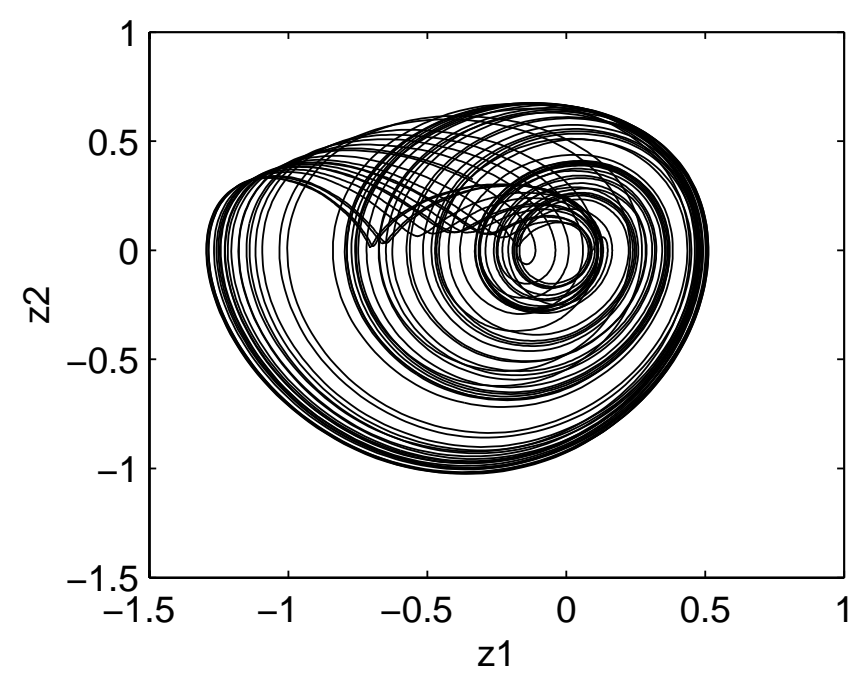

(b)

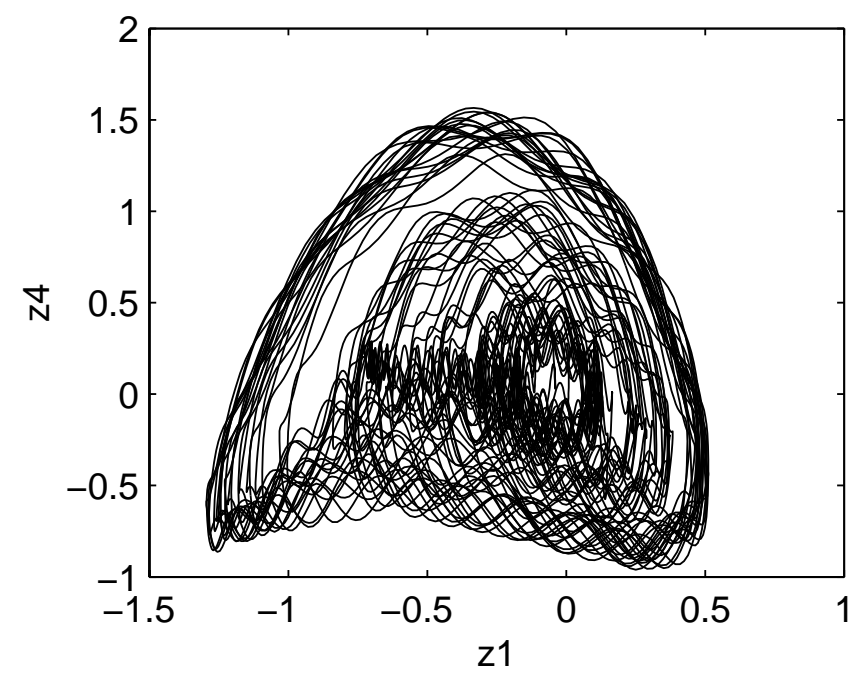

(d)

Fig. 2. Simulation result for flexible beam: nonideal case, (a) control input $u$, (b) $z_{1}$ versus $z_{2}$, (c) $z_{1}$ versus $z_{3}$, (d) $z_{1}$ versus $z_{4}$.

the same equations with the modifications given above. The results are shown in Fig. 2. Here, the control input $u$ is shown in Fig. 2(a), and $z_{1}$ versus $z_{2}, z_{3}$ and $z_{4}$ are shown in Figs. 2(b) $-2(\mathrm{~d})$, respectively. Note that the perturbations, especially the noise level, is not low, nevertheless the system still exhibits chaotic behavior.

\section{Conclusion}

In this paper, we considered a model-based approach to the anticontrol of some continuous-time systems, where our aim was to generate chaotic behavior in a given system by means of an appropriate control input. We assumed the existence of a reference chaotic model in an appropriate form. Then we determined an appropriate control input to match the dynamics of the system to be controlled with that of the model chaotic system. We proved that: (i) any controllable linear time-invariant system can be chaotized with an appropriate input, (ii) this approach could be generalized to a class of nonlinear systems. We proposed a simple procedure to generate such chaotic models in arbitrary dimension. We also considered the computability of the required feedback law by using only the available signals. To estimate the states of the system to be controlled, we proposed an observer-based synchronization scheme. Under some mild conditions, exponentially fast synchronization may be achieved, 
and one can use the estimated states to compute the feedback law. We also commented on the robustness of the proposed scheme.

Note that the control inputs given by (9) or (12) will remain bounded provided that the solutions of (4) or (11) remain bounded. This condition is satisfied in chaotic systems, since chaotic solutions have a domain of attraction. However, other than this boundedness, nothing can be claimed in our approach on the bound of the control input, e.g. we cannot claim that it could be made arbitrarily small. The optimization of the control input is not considered in our work. This topic has practical importance which deserves further investigation.

\section{References}

Boccaletti, S., Grebogi, C., Lai, Y. C., Mancini, H. \& Maza, D. [2000] "The control of chaos: Theory and applications," Phys. Rep. 329, 103-197.

Brandt, M. E. \& Chen, G. [1997] "Bifurcation control of two nonlinear models of cardiac activity," IEEE Trans. Circuits Syst.-I 44, 1031-1034.

Chen, G. \& Moiola, J. L. [1994] "An overview of bifurcation, chaos, and nonlinear dynamics in nonlinear systems," J. Franklin Inst. B331, 819-858.

Chen, G. [1996] "Control and synchronization of chaotic systems, (a bibliography)," ftp.egr.uh.edu/ pub/TeX/chaos.tex

Chen, G. \& Lai, D. [1996] "Feedback control of Lyapunov exponents for discrete-time dynamical systems," Int. J. Bifurcation and Chaos 6, 1341-1349.

Chen, G. \& Dong, X. [1998] From Chaos to Order: Methodologies, Perspectives and Applications (World Scientific, Singapore).

Chen, G. \& Lai, D. [1998] "Feedback anticontrol of discrete chaos," Int. J. Bifurcation and Chaos 8, $1585-1590$.

Ditto, W. L. [1996] "Applications of chaos in biology and medicine," in Chaos and the Changing Nature of Science and Medicine: An Introduction, ed. Herbert, D. E. (AIP Press, NY), pp. 175-201.

Fradkov, A. L. \& Pogromsky, A. Y. [1998] Introduction to Control of Oscillations and Chaos (World Scientific, Singapore).

Fradkov, A. L. \& Evans, R. J. [2002] "Control of chaos: Survey 1997-2000," Proc. IFAC 2002 World Congress, July 2002, Barcelona, Spain.

Genesio, R. \& Tesi, A. [1992] "Harmonic balance methods for the analysis of chaotic dynamics in nonlinear systems," Automatica 28, 531-548.

Goldberger, A. L. [1994] "Applications of chaos to physiology and medicine," in Applied Chaos, eds. Kim, J. H. \& Stringer, J. (Academic Press, NY), pp. $321-331$.

Isidori, A. [1995] Nonlinear Control Systems, 3rd edition (Springer-Verlag, Berlin).

Kailath, T. [1980] Linear Systems (Prentice-Hall, NJ).

Kapitaniak, T. [2000] Chaos for Engineers, Theory, Applications and Control (Springer-Verlag, Berlin).

Morgül, Ö. \& Solak, E. [1996] "On the observer based synchronization of chaotic systems," Phys. Rev. E54, 4803-4811.

Morgül, Ö. \& Feki, M. [1997] "Synchronization of chaotic systems by using occasional coupling," Phys. Rev. E55, 5004-5010.

Morgül, Ö. \& Solak, E. [1997] "On the synchronization of chaotic systems by using state observers," Int. J. Bifurcation and Chaos 7, 1307-1322.

Morgül, Ö. [1999a] "Necessary condition for observerbased chaos synchronization," Phys. Rev. Lett. 82, 169-176.

Morgül, Ö. [1999b] "On the control of some chaotic systems by using dither," Phys. Lett. A262, 144-151.

Morgül, Ö., Solak, E. \& Akgül, M. [2002] "Observer based chaotic message transmission," Int. J. Bifurcation and Chaos 13, 1003-1017.

Ott, E., Grebogi, C. \& Yorke, J. A. [1990] "Controlling chaos," Phys. Rev. Lett. 64, 1196-1199.

Schiff, S. J., Jerger, K., Duang, D. H., Chang, T., Spano, M. L. \& Ditto, W. L. [1994] "Controlling chaos in the brain," Nature 370, 615-620.

Shinbrot, T., Ott, E., Grebogi, C. \& Yorke, J. A. [1990] "Using chaos to direct trajectories to targets," Phys. Rev. Lett. 65, 3215-3218.

Solak, E., Morgül, Ö. \& Ersoy, U. [2001] "Observerbased control of a class of chaotic systems," Phys. Lett. A279, 47-55.

Vidyasagar, M. [1993] Nonlinear Systems Analysis, 2nd edition (Prentice-Hall, NJ).

Wang, X. F. \& Chen, G. [2000] "Chaotifying a stable LTI system by tiny feedback control," IEEE Trans. Circuits Syst.-I 47, 410-415. 